antigen has a favorable expression profile with absence in vital normal human tissues. In this study, we sought to establish and validate clinical-grade manufacturing of ROR1 CAR$\mathrm{T}$ to enable a Phase $\mathrm{I} / \mathrm{II}$ a clinical trial. In particular, we sought to integrate virus-free gene-transfer based on Sleeping Beauty transposition into this manufacturing protocol to permit scale-up and export to point-of-care manufacturing, and to reduce turn-around time, complexity and regulatory burden associated with conventional viral gene-transfer (biosafety level 2 to biosafety level 1 ).

Materials and Methods Buffy coats or leukaphereses were obtained from healthy donors to perform protocol optimization $(n=7)$ and scale-up runs $(n=1) . \mathrm{CD}^{+}$and $\mathrm{CD}^{+}$T cells were isolated separately by magnetic selection and stimulated with CD3/CD28 TransACT ${ }^{\circledR}$ reagent. T cells were transfected with mRNA encoding hyperactive Sleeping Beauty transposase (SB100X) and minicircle DNA (MC) encoding a pT2 transposon comprising the ROR1 CAR and an EGFRt marker gene using the MaxCyte GTx ${ }^{\circledR}$ electroporation platform. Following transfection, $\mathrm{T}$ cells were expanded for 10-13 days in GREX $^{\circledR}$ bioreactors and then harvested and formulated into the drug product at a 1:1 ratio of CAR-expressing CD4:CD8 $\mathrm{T}$ cells. The drug product underwent comprehensive phenotypic, functional and genomic analyses as part of product qualification.

Results The set of protocol optimization runs resulted in a highly robust process. On average, the stable gene-transfer rate at the end of the manufacturing process was $71 \%$ in CD4+ $(n=5)$ and $54 \%$ in $\mathrm{CD}^{+} \mathrm{T}$ cells $(\mathrm{n}=7)$. The average yield of ROR1 CAR-T relative to the number of input $\mathrm{T}$ cells was 12.6-fold for $\mathrm{CD}^{+}{ }^{+}$and 9.4-fold for $\mathrm{CD} 8^{+}$after $12-15$ days of expansion, with an average viability of $84 \%$ for $\mathrm{CD}^{+}$and $82 \%$ of $\mathrm{CD} 8^{+} \mathrm{T}$ cells. The scale-up run was performed with a leukapheresis product from which $52.5 \times$ $10^{\wedge} 6 \mathrm{CD}^{+}$and $109 \times 10^{\wedge} 6 \mathrm{CD}^{+} \mathrm{T}$ cells were transfected. At the end of the manufacturing process (day 12), there were $844 \times 10^{\wedge} 6$ CAR-expressing CD4 ${ }^{+}(\sim 16$-fold expansion) and $857 \times 10^{\wedge} 6$ CAR-expressing CD ${ }^{+} \mathrm{T}$ cells (8-fold expansion). In functional testing, ROR1 CAR-T showed specific recognition and potent elimination of $\mathrm{ROR}^{+}{ }^{+}$target cells, as well as antigen-dependent cytokine production and productive proliferation in in vitro analyses. Experiments to determine the anti-tumor potency of the drug product in vivo and detailed genomic analyses are ongoing. Preliminary analyses suggest a favorable genomic insertion profile of the CAR transposon, and a transposon copy number that is well within the range acceptable for clinical use of the drug product.

Conclusions With this novel protocol, we aim to obtain the first manufacturing license for CAR-T in Europe that integrates our optimized approach with SB100X mRNA and transposon MC for CAR gene-transfer on the MaxCyte transfection platform. The quality and yield of the drug product support the design and dose escalation of the proposed clinical trial with ROR1 CAR-T, and will serve as a blueprint for other CAR-T products from our pipeline.

Disclosure Information K. Mestermann: None. M. Eichler: None. M. Machwirth: None. K. Kebbel: None. U. Köhl: None. H. Einsele: None. C. Müller: None. J. Lehmann: None. T. Raskó: None. F. Lundberg: None. Z. Izsvák: None. G. Schmiedeknecht: None. M. Hudecek: None.

\section{P09.09 PD-1 CHECKPOINT BLOCKADE FOR TREATMENT OF MUCORMYCOSIS AND INVASIVE ASPERGILLOSIS IN A STEM CELL TRANSPLANT RECIPIENT}

1,2 $\mathrm{N}$ Mueller*, 'J Banck, ${ }^{3} \mathrm{~S}$ Mellinghoff, ${ }^{4,5} \mathrm{H}$ Schlösser, ${ }^{4,5} \mathrm{M}$ Thelen, ${ }^{3} \mathrm{P}$ Koehler, ${ }^{6} \mathrm{~F}$ Schrötzlmair, ${ }^{3} \mathrm{O}$ Cornely, ${ }^{1} \mathrm{LH}$ Lindner, ${ }^{1,7,8} \mathrm{M}$ von Bergwelt-Baildon. ${ }^{1}$ Department of Medicine III, University Hospital, LMU Munich, Munich, Germany; 'Laboratory for Translational Cancer Immunology, Gene Center, LMU Munich, Munich, Germany; ${ }^{3}$ University of Cologne, Faculty of Medicine and University Hospital Cologne, Department I of Internal Medicine, Excellence Center for Medical Mycology (ECMM), Cologne, Germany; ${ }^{4}$ Center for Molecular Medicine Cologne, University of Cologne, Faculty of Medicine and University Hospital Cologne, Cologne, Germany; ${ }^{5}$ Department of General, Visceral, Cancer and Transplantation Surgery, University of Cologne, Faculty of Medicine and University Hospital Cologne, Cologne, Germany; ${ }^{6}$ Department of Otorhinolaryngology, Head and Neck Surgery, University Hospital, LMU Munich, Munich, Germany; ${ }^{7}$ German Cancer Consortium (DKTK), partner site Munich, Munich, Germany; ${ }^{8}$ Work group iCHOP (Intensive Care in Hematologic and Oncologic Patients), Berlin, Germany

\subsection{6/jitc-2020-ITOC7.109}

Background Despite early surgical debridement and application of systemic antifungal drugs, invasive fungal infections by mucor spp. are still associated with a very poor prognosis in immunocompromised patients. Due to their lack of immune defense, targeted treatment strategies reversing the hyporesponsiveness of the immune system by immune checkpoints might improve patients' outcome. Until today, a successful recovery of mucormycosis after receiving anti-PD-1 antibody is only described once for a polytrauma patient. Therefore, we here describe the first immunosuppressed patient treated with nivolumab for invasive mucormycosis with aspergillus coinfection.

Materials and Methods A 51-year-old woman from Germany with acute myeloid leukemia (AML) relapse after allogenic hematopoietic stem cell transplantation was treated with azacitidine and lenalidomide. She acquired an invasive fungal infection with mucor species Lichtheimia ramosa combined with Aspergillus fumigatus in functional pancytopenia. Three surgical pansinusrevisions were performed and high dose i.v. antifungal treatment with liposomal amphotericin $B$ and isavuconazole was initiated. Due to missing treatment response with daily mucor progression nivolumab $240 \mathrm{mg}$ was administered and complemented by interferon $\gamma(100 \mu \mathrm{g}$ s.c. 5 doses). Administration was repeated every 2 weeks (in total 4 doses of nivolumab, but only 10 doses of interferon $\gamma$ due to recurrent fever episodes) and simultaneously i.v. antifungal treatment was deescalated. Blood samples were collected before (baseline treatment (BT)) as well as 2 weeks (under treatment (UT) 1) and 5 weeks (UT2) after treatment initiation with nivolumab. Peripheral blood mononuclear cells were isolated and flow cytometry analyses of lymphocytic subsets were performed.

Results Ten days after first dose of nivolumab, long-term local hemostasis was achieved. Local symptoms disappeared, sinusitis complaints improved, and inflammation values decreased significantly. Sixteen days after treatment initiation a CT scan revealed a partial remission of mucormycosis invasion. Follow-up CT scans showed a stable disease. Expression of PD-1 on T cells was monitored as proof of concept from BT on and showed a significant reduction from $34.7 \%$ to $3.3 \%$ (UT1) and $1.38 \%$ (UT2). Both activation markers CD86 and CD69 showed an increase from BT to UT1. T cells showed high maturation markers throughout monitoring, while B cell maturation increased from BT to UT1/2. Nine 
weeks after diagnosis and despite long-term neutropenia the patient was still clinically stable under nivolumab treatment and discharged with continued deescalated antimycotic treatment. A bone marrow biopsy revealed a further progression of AML relapse. After 3 weeks during follow-up mucormycosis was still clinically stable. Ten days later the patient developed fever up to $39.5^{\circ} \mathrm{C}$, but refused to seek medical attention due to unfavorable prognosis of AML and died two days later from septic shock combined with disseminated intravascular coagulation.

Conclusions In immunocompromised hematological patients with invasive fungal infections, immune checkpoint inhibition is capable of reversing an infection-induced immunosuppressive phenotype. Therefore, it might complement the treatment of invasive fungal infections and should be evaluated in future clinical trials.

Disclosure Information N. Mueller: None. J. Banck: None. S. Mellinghoff: None. H. Schlösser: None. M. Thelen: None. P. Koehler: None. F. Schrötzlmair: None. O. Cornely: None. L. H. Lindner: None. M. von Bergwelt-Baildon: None.

\section{P09.10 LOCAL IMMUNOTHERAPY OF BRAIN CANCER HARNESSING HIGH-RETENTION FC-FUSION CONSTRUCTS}

L Schellhammer*, M Beffinger, S Pantelyushin, T Buch, J vom Berg. Institute of Laboratory Animal Science, University of Zurich, Zurich, Switzerland

\subsection{6/jitc-2020-ITOC7.110}

Background Glioblastoma is a highly aggressive cancer type and despite aggressive therapy, patients' survival remains poor. Immunotherapy of brain cancer is particularly difficult because of its location behind the blood-brain-barrier and the immunosuppressive tumour microenvironment. In order to (re-)activate the immune system, and reverse the local immunosuppression, we employ the pro-inflammatory cytokine interleukin 12 (IL12). This highly potent immune-stimulatory agent is known for its anti-cancer effect. Unfortunately, IL-12 was found to induce severe toxicity when applied intravenously, impeding its way into clinics. Thus, currently the only valid therapeutic option is local application into the tumour site.

Materials and Methods Engineered proteins were expressed in HEK293T cells and purified by affinity chromatography. In vivo experiments were performed in glioma-bearing mice using intracranial injection of bioluminescent GL-261 cell line. Treatments were performed on day 21 and 28 post tumour injection through intracranial injection using a step-catheter modelling convection enhanced delivery in mice. Blood or tissue was analysed using immunohistochemistry, flow cytometry and ELISA.

Results Based on an IL-12-IgG fusion protein, we engineered a molecule for exclusively local therapy of brain cancer. We showed anti-cancer efficacy and increased tissue retention of the fusion molecule in glioma in mice. However, molecular analysis of treated tissue confirmed an upregulation of the immunosuppressive molecule PD-L1 in the tumour microenvironment. This means that, despite its efficacy, IL-12 induces an adaptive resistance mechanism, counteracting the therapeutic effect. We thus hypothesised that local IL-12 therapy combined with local blockade of the PD-1/PD-L1-axis would further improve therapeutic efficacy, while exclusively local administration would avoid increased side effects, which usually accompany combination immunotherapy. We showed significantly enhanced long-term survival of glioma-bearing mice treated with IL-12 therapy in combination with PD-L1 blockade compared to single or control treatments. In a next step, we engineered a novel, bifunctional molecule. Optimized for local application and minimized leakage into the systemic circulation, it combines immune-stimulation and checkpoint blockade in one entity. We showed anti-cancer efficacy and increased tissue retention in glioma in mice.

Conclusions The potent anti-cancer effect of the cytokine IL12 can be used in therapy when applied locally into the brain tumour. Besides fusion to $\operatorname{IgG}$, we introduced several specific modifications on the molecule, which are crucial to prevent systemic exposure and associated toxic side effects. To overcome the dampening of the immune reaction through induced PD-L1 expression, we introduced a combination therapy of IL-12 with a PD-L1-blocking antibody in a single molecule. We showed this combination superior to single treatments in the context of exclusively local brain tumour therapy.

Disclosure Information L. Schellhammer: None. M. Beffinger: None. S. Pantelyushin: None. T. Buch: None. J. vom Berg: None.

\section{P09.11 TLR3 SUPPRESSES COLORECTAL CARCINOGENESIS, PRESUMABLY THROUGH UP-REGULATION OF T-CELL ATTRACTING CXC CHEMOKINES}

A Sichler*, M Frey, W Johannes, K Janssen. Klinikum rechts der Isar TUM, München, Germany

\subsection{6/jitc-2020-ITOC7.111}

Background Toll-like-receptors (TLRs) are main components of the innate immune system which recognize endogenous or pathogen-associated molecular 'danger patterns'. Previous findings from us and others highlighted a role of TLRs in the formation of tumors. However, TLRs may have differing roles in immune and cancer cells, and the underlying mechanisms are still unclear. TLR downstream signaling is mediated by two adapter proteins; MyD88 (myeloid differentiation primary response gene 88) and TRIF (TIR-domain-containing adapter-inducing interferon- $\beta$ ). The MyD88-mediated signaling pathway is activated by all TLRs, except TLR3. We could show previously that it leads to the initiation of oncogenic, proliferative and pro-inflammatory responses in colorectal cancer. The endosomal receptor TLR3, in contrast, solely depends on TRIF. It recognizes viral, microbial and endogenous dsRNA leading to production of type-I interferon and chemokines, and induces apoptosis. The role of TRIF dependent TLR3 signaling in colorectal cancer is still disputed. Within this study, we show tumor-suppressive TLR3 functions prevailing over oncogenic effects in colorectal cancer.

Materials and Methods TLR3-deficient colon cancer cell lines were engineered by CRISPR-Cas9. Genetically modified mouse models were generated, based on a 'switch-on mutagenesis' approach, with global inactivation of Tlr3 or TRIF (Ticam1), allowing tissue specific re-expression based on Cre recombination. The mice were interbred with the $\mathrm{Apc}^{1638 \mathrm{~N}}$ mouse model for digestive cancer. Furthermore, clinical significance of TLR3 expression levels was assessed in human colorectal cancer tissue samples from our clinic $(n=81)$ and from TCGA datasets. A putative correlation between 